\title{
Efficacy of intravenous sedation and oral nifedipine in dental implant patients with preoperative hypertension - a retrospective study of 516 cases
}

Motoshi Kimura' ${ }^{1 *}$, Yoshihiro Takasugi ${ }^{2}$, Shigeyoshi Hanano ${ }^{1}$, Katsuyuki Terabe ${ }^{3}$ and Yuko Kimura ${ }^{4}$

\begin{abstract}
Background: To examine the effects of intravenous sedation and oral nifedipine on blood pressure and pulse rate in patients with perioperative high blood pressure undergoing implant surgery, the clinical records of dental implant patients managed by intravenous sedation at our outpatient dental offices were retrospectively evaluated.

Methods: A total of 516 clinical charts were evaluated. The subjects were divided into two groups: a normotensive group with no history of hypertension and a hypertensive group with a history of hypertension. The patients in the hypertensive group were further divided into two subgroups: with or without nifedipine administration before operation. Systolic blood pressure (SBP), diastolic blood pressure (DBP), pulse rate (PR), and rate pressure product (RPP) were assessed.

Results: In 30 patients (33\%) of the hypertensive group, the high blood pressure on arrival obviously declined to around or less than $160 \mathrm{mmHg}$; in the remaining patients in the group who showed a mean SBP of $182.1 \pm 13.8 \mathrm{mmHg}$ on arrival, the blood pressure did not decrease after a 30-min rest. Oral nifedipine administered to the patients with sustained high blood pressure decreased SBP to $144.7 \pm 23.1 \mathrm{mmHg}$ in $28.1 \pm 9.3 \mathrm{~min}$ after administration, comparable to that in hypertensive patients without nifedipine.

Conclusions: For patients with stage 2 hypertension before operation, it may be difficult to maintain the recommended blood pressure during surgery by only intravenous sedation; reduction of blood pressure by antihypertensive drugs may be necessary.
\end{abstract}

Keywords: Oral surgery; Dental implant; Hemodynamics; Intravenous sedation; Nifedipine administration

\section{Background}

Osseointegrated dental implants were introduced in Japan in 1983, and the procedures are now performed very frequently. Dental implants are placed in a wide age range of patients, including elderly patients with hypertension. Patients with very high blood pressure are at great risk for acute medical problems when undergoing stressful dental procedures, such as oral surgery, periodontal surgery, and placement of dental implants [1].

Patients with normal blood pressure $(<120 / 80 \mathrm{mmHg})$, prehypertension (120 to $139 / 80$ to $89 \mathrm{mmHg}$ ), or stage I hypertension (140 to $159 / 90$ to $99 \mathrm{mmHg}$ ) may receive

\footnotetext{
* Correspondence: kimkimkim0719@yahoo.co.jp

'Hanano Dental Clinic, 4-2-3 Yamanoue, Hirakata, Osaka 573-0047, Japan Full list of author information is available at the end of the article
}

regular dental care, but those with stage 2 hypertension $(\geq 160 / \geq 100 \mathrm{mmHg})$ should receive noninvasive treatment only and be referred to the physician for immediate follow-up [2]. Normotensive individuals may develop signs of hypertensive encephalopathy at blood pressures as low as $160 / 100 \mathrm{mmHg}$, whereas chronically hypertensive patients can tolerate higher blood pressure and may not do so until the blood pressure rises to $220 / 110 \mathrm{mmHg}$ or above [3]. Although no recommendation has been presented on the optimal level of blood pressure to avoid hypertensive complications during invasive dental treatments, blood pressure in hypertensive patients should be maintained below 160/100 mmHg.

Pain, stress, or anxiety-related dental procedures can raise blood pressure in both hypertensive and normotensive 
patients [4]. We have employed intravenous sedation to manage patients with hypertension as well as dental anxiety and phobia. The oral antihypertensive agent nifedipine is mainly administered to patients with high systolic blood pressure (SBP) $\geq 160 \mathrm{mmHg}$ prior to implant placement. To examine the effects of intravenous sedation and oral nifedipine on blood pressure and pulse rate in patients with perioperative high blood pressure, the clinical records of dental implant patients managed by intravenous sedation at our outpatient dental offices were retrospectively evaluated. The purpose of this clinical study is to examine whether intravenous sedation and oral administration of nifedipine is efficient for the hemodynamic for the patient with hypertension. The authors expect that it is possible not only to obtain a hemodynamic blood stable but also to prevent the medical sequelae by performing intravenous sedation and oral nifedipine for patients with hypertension.

\section{Methods}

A retrospective review of the clinical records was conducted for 336 patients who received dental implantrelated surgeries combined with intravenous sedation between January 2008 and February 2012 at our outpatient dental offices. Among the patients, 125 patients received multiple surgeries during the observation period: 4 patients underwent surgery five times, 7 patients four times, 29 patients three times, 85 patients twice, and others once. The following surgical procedures were performed in a total of 516 patients: dental implant placement (466 patients), sinus lift surgery and dental implant (28 patients), socket lift and dental implant (10 patients), and guided bone regeneration and dental implant (12 patients).

We performed surgeries after medical consultation when patients had a history of hypertension or cardiovascular or cerebrovascular diseases. History of ischemic heart disease, renal dysfunction, diabetes mellitus, cerebral infarction, or articular rheumatism was documented in 17 patients in the hypertensive group and 16 patients in the normotensive group. They were confirmed stable and well controlled for implant surgery. For patients who received therapeutic drugs, surgery was performed following daily medication.

The patients were allowed to have water or snacks until $2 \mathrm{~h}$ before the visit. On arrival at the office, systolic blood pressure (SBP), diastolic blood pressure (DBP), and pulse rate (PR) were measured using an automatic blood pressure monitor with an oscillometric method (HEM-1010, Omron Healthcare, Kyoto, Japan). The cephalic vein was cannulated with a $22-\mathrm{G}$ disposable intravenous catheter. Nifedipine capsule $(10 \mathrm{mg}$ ) was orally administered to patients with sustained increases in $\mathrm{SBP} \geq 160 \mathrm{mmHg}$ for $30 \mathrm{~min}$ from baseline measurement. A noninvasive blood pressure monitoring system with electrocardiogram (ECG) monitor and pulse oximeter (Moneo
BP-88, Omron Healthcare, Kyoto, Japan) was mounted, and blood pressure was measured at 2- to 5-min intervals. In patients with a history of cardiovascular disease, ECG was continuously monitored. Following confirmation of a definite decline of blood pressure or SBP $<160 \mathrm{mmHg}$, infiltration anesthesia and/or conduction anesthesia was administered using 1 to 3 cartridges ( 1.8 to $5.4 \mathrm{ml}$ ) of $2 \%$ lidocaine containing 1/80,000 epinephrine.

Following confirmation of a sufficient anesthetic effect, intravenous sedation with continuous infusion of propofol 1 to $2 \mathrm{mg} / \mathrm{kg} / \mathrm{h}$ and midazolam 20 to $40 \mu \mathrm{g} / \mathrm{kg}$ bolus together with inhalation of oxygen $3 \mathrm{~L} / \mathrm{min}$ via nasal cannula was initiated. After confirming Verrill sign, implant surgery was initiated. During operation, the propofol dose was adjusted to maintain the optimum conscious sedative condition (level 2 on the Ramsay sedation scale) [5], and local anesthesia was added when the patient complained of pain. On completion of surgery, administration of oxygen and propofol were terminated and the patient was observed for about $1 \mathrm{~h}$, until normal cognitive and motor functions were restored.

The subjects were divided into two groups: a normotensive group with no history of hypertension and a hypertensive group with a history of hypertension. Thirteen patients who had no history of hypertension were included in the hypertensive group, since they indicated SBP $\geq 160 \mathrm{mmHg}$ on arrival at the office and were later diagnosed with essential hypertension by cardiologists. Furthermore, the patients in the hypertensive group were divided into two subgroups: with or without nifedipine administration.

From the clinical chart, data of SBP, DBP, PR, and percutaneous oxygen saturation $\left(\mathrm{SpO}_{2}\right)$ were sampled at the point of arrival to the office, prior to the initiation of sedation, $30 \mathrm{~min}$ after the initiation of operation, and on completion of operation. Furthermore, rate pressure product $(\mathrm{RPP}: \mathrm{SBP} \times \mathrm{PR})$ was calculated.

There were two primary outcome measures: (1) incidence of improved hypertension following oral nifedipine and (2) incidence of normal ranges of hemodynamic parameters during surgery. The secondary outcome variable was incidence of hypertension related to perioperative complications.

\section{Statistical analysis}

In this study, we used data from all cases (516 cases) for statistical analysis.

Data were described as mean \pm standard deviation. The unpaired $t$ test was used to compare demographic variables between groups. Fisher's exact test was used to compare ratios of patients in hypertensive group between subgroups. One-way analysis of variance (ANOVA) followed by Tukey's multiple comparison test was performed to examine the change in the values of parameters. Repeated measures 
ANOVA followed by Dunnett's multiple comparison test was used to compare the values of parameters in groups at each time point. Statistical analysis was performed using Prism 5 for Windows Ver. 5.01 (GraphPad Software Inc., San Diego, CA, USA). The significance level was set at $p<0.05$.

\section{Ethical approval}

This study protocol was approved by the ethics committee of Japanese Dental Society of Anesthesiology (No. 2015-4).

\section{Results}

Patient demographics and clinical characteristics are summarized in Table 1 . There were significant differences in age $(p<0.0001)$ and duration of surgery $(p=0.025)$ between normotensive and hypertensive groups. On arrival at the office, values of all hemodynamic parameters, including SBP, DBP, PR, and RPP, were higher in the hypertensive group than in the normotensive group $(p<0.0001)$.

Tables 2 and 3 indicate incidences of patients with SBP $\geq 160 \mathrm{mmHg}$ and RPP $\geq 12,000 \mathrm{bpm} \times \mathrm{mmHg}$, and perioperative hemodynamic changes, respectively. On arrival at the office, 66 patients (62\%) in the hypertensive group and 41 patients (10\%) in the normotensive group revealed high SBP $\geq 160 \mathrm{mmHg}$. Thirty minutes later, SBP declined to less than $160 \mathrm{mmHg}$ in most of the patients in the normotensive group. On the other hand, in 30 patients (33\%) in the hypertensive group, high blood pressure on arrival obviously declined to around or less than $160 \mathrm{mmHg}$, while in the remaining patients in the group who showed a mean SBP of $182.1 \pm 13.8 \mathrm{mmHg}$ on arrival, the blood pressure did not clearly decrease after $30 \mathrm{~min}$ of rest. Oral nifedipine administered to patients with sustained high blood pressure decreased SBP to $144.7 \pm 23.1 \mathrm{mmHg}$ by $28.1 \pm 9.3$ min after administration, which was similar to that in hypertensive patients without nifedipine.
Although the mean SBP in the hypertensive group was significantly higher than that in the normotensive group during operation, SBP $<160 \mathrm{mmHg}$ was maintained in all patients except three in the hypertensive group (2.8\%) and one in the normotensive group $(0.2 \%)$. The values of DBP in patients in the hypertensive group were higher than those in the normotensive group throughout the observation course, and changes in DBP in each group were similar to those in SBP.

On arrival at the office, $\mathrm{RPP} \geq 12,000 \mathrm{bpm} \times \mathrm{mmHg}$ was found in 74 patients $(70 \%)$ in the hypertensive group and 111 patients $(27 \%)$ in the normotensive group. More than $90 \%$ of the patients with preoperative nifedipine showed high RPP on arrival at the office. Among patients with high RPP, all patients in the normotensive group, $10 \%$ of the patients without nifedipine in the hypertensive group and $35 \%$ of patients with nifedipine in the hypertensive group had $\mathrm{RPP}<12,000 \mathrm{bpm} \times \mathrm{mmHg}$ until initiation of intravenous sedation. The values of RPP during operation under intravenous sedation were maintained at a normal range except in nine patients $(8.5 \%)$ in the hypertensive group and one patient $(0.2 \%)$ in the normotensive group.

In patients with oral nifedipine in the hypertensive group, the PR value slightly increased prior to initiation of intravenous sedation $(p=0.224)$ and then significantly decreased until completion of the operation $(p<0.001)$.

All patients stated a pleasant feeling and amnesia during surgery. No complication occurred during surgery, and no cognitive and motor dysfunctions were observed $1 \mathrm{~h}$ after surgery. The patients revealed SBP of $>160 \mathrm{mmHg}$ during and at completion of operation showed maximum SBP of $180 \mathrm{mmHg}$ in the normotensive group, $190 \mathrm{mmHg}$ on the hypertensive group without preoperative oral nifedipine, and $180 \mathrm{mmHg}$ in the hypertensive group with preoperative oral nifedipine. They did not complaint any symptom such as headache, confusion, and chest pain. Upon leaving

Table 1 Demographic and clinical characteristics

\begin{tabular}{llll}
\hline & Normotensive group & Hypertensive group & $p$ value \\
\hline Number (male: female) & $410(127: 283)$ & $106(37: 69)$ & 0.170 \\
Age (year mean \pm SD) & $59 \pm 11$ & $65 \pm 10$ & $<.0001$ \\
Weight $(\mathrm{kg})$ & $54.5 \pm 9.2$ & $57.1 \pm 10.9$ & \\
Values of circulation parameters on arrival at office & & & \\
$\quad$ SBP (mmHg) & $133 \pm 19$ & $165 \pm 22$ & $<04$ \\
DBP (mmHg) & $76 \pm 13$ & $99 \pm 20$ & $<0.0001$ \\
PR (bpm) & $79 \pm 13$ & $90 \pm 19$ & $<.0001$ \\
RPP (bpm $\times$ mmHg) & $10,574 \pm 2,614$ & $12,367 \pm 5,771$ & $<0.0001$ \\
Preoperative oral nifedipine & 0 & $44(42 \%)$ & $<0.0001$ \\
Duration of surgery (min) & $40 \pm 20$ & $35 \pm 17$ & 0.025 \\
Duration of sedation (min) & $69 \pm 25$ & $65 \pm 22$ & 0.114 \\
\hline
\end{tabular}

SBP, systolic blood pressure; DBP, diastolic blood pressure; PR, pulse rate; RPP. rate pressure product. 
Table 2 Incidence of high blood pressure and high rate pressure product

\begin{tabular}{lll}
\hline & SBP $(>\mathbf{1 6 0} \mathbf{~ m m H g})$ & $\mathbf{R P P}(>\mathbf{1 2 , 0 0 0} \mathbf{~ b p m ~} \times \mathbf{~ m m H g})$ \\
\hline Normotensive group $(N=410)$ & $41(10.0 \%)$ & $111(27.1 \%)$ \\
On arrival at the office & $31(7.6 \%)$ & $72(17.6 \%)$ \\
Prior to sedation & $1(0.2 \%)$ & $1(0.2 \%)$ \\
During operation & $3(0.7 \%)$ & $9(2.2 \%)$ \\
Completion of operation & $22(35.5 \%)^{* *}$ & $34(54.8 \%)^{* *}$ \\
Hypertensive group without preoperative oral nifedipine $(N=62)$ & $27(43.5 \%)^{* *}$ \\
On arrival at the office & $13(21.0 \%)^{* *}$ & $5(8.1 \%)^{* *}$ \\
Prior to sedation & $2(3.2 \%)^{*}$ & $6(9.7 \%)^{* *}$ \\
During operation & $5(8.1 \%)^{* *}$ & $40(90.9 \%)^{* *}$ \\
Completion of operation & $44(100 \%)^{* *}$ & $24(54.5 \%)^{* *}$ \\
Hypertensive group with preoperative oral nifedipine $(N=44)$ & $4(18.2 \%)^{*}$ & $4(9.1 \%)^{* *}$ \\
On arrival at the office & $1(2.2 \%)$ & $8(18.2 \%)^{* *}$ \\
Prior to sedation & $3(6.8 \%)^{*}$ & \\
During operation & & \\
Completion of operation &
\end{tabular}

${ }^{*} p<0.05,{ }^{* *} p<0.01$ vs normotensive group (Fisher's exact test). SBP, systolic blood pressure; RPP, rate pressure product.

the office, high SBP of the patients decreased to the level on arrival without any antihypertensive treatment.

\section{Discussion}

In $44(8.5 \%)$ of the 516 implant surgery cases, oral nifedipine had to be administered, since preoperative SBP was higher than $160 \mathrm{mmHg}$ in these patients. Within 30 min of administration of nifedipine, SBP of hypertensive patients decreased to a similar range as that of hypertensive patients who did not need administration of oral nifedipine. Intravenous sedation after nifedipine administration to hypertensive patients resulted in stable hemodynamics during implant surgery.

The Seventh Report of the Joint National Committee on Prevention, Detection, Evaluation, and Treatment of High Blood Pressure [6] classified hypertensive patients into five categories based on systolic or diastolic blood pressure. Patients with normal blood pressure $(<120 / 80 \mathrm{mmHg})$, prehypertension (120 to $139 / 80$ to $89 \mathrm{mmHg}$ ), or stage I hypertension (140 to $159 / 90$ to $99 \mathrm{mmHg}$ ) can receive

Table 3 Changes in values of hemodynamic parameters

\begin{tabular}{|c|c|c|c|c|}
\hline & $\mathrm{SBP}(\mathrm{mmHg})$ & $\mathrm{DBP}(\mathrm{mmHg})$ & PR (bpm) & $\mathrm{RPP}(\mathrm{bpm} \times \mathrm{mmHg})$ \\
\hline \multicolumn{5}{|c|}{ Normotensive patients $(N=410)$} \\
\hline On arrival at the office & $133.0 \pm 18.4$ & $76.4 \pm 12.5$ & $79.2 \pm 13.4$ & $10,603 \pm 2,623$ \\
\hline Prior to sedation & $128.5 \pm 18.3^{*}$ & $70.5 \pm 11.9^{*}$ & $76.8 \pm 13.0^{*}$ & $9,927 \pm 2,485^{*}$ \\
\hline During operation & $109.7 \pm 11.9^{*}$ & $62.7 \pm 10.0^{*}$ & $70.6 \pm 10.2^{*}$ & $7,768 \pm 1,555^{*}$ \\
\hline Completion of operation & $115.9 \pm 14.2^{*}$ & $66.1 \pm 11.3^{*}$ & $70.4 \pm 10.7^{*}$ & $8,196 \pm 1,796^{*}$ \\
\hline \multicolumn{5}{|c|}{ Hypertensive patients without preoperative oral nifedipine $(N=62)$} \\
\hline On arrival at the office & $152.5 \pm 17.6$ & $88.3 \pm 11.8$ & $84.6 \pm 14.3$ & $12,972 \pm 3,055$ \\
\hline Prior to sedation & $143.2 \pm 18.1^{*}$ & $77.4 \pm 9.6^{*}$ & $82.7 \pm 13.4$ & $11,874 \pm 2,540^{*}$ \\
\hline During operation & $118.9 \pm 15.5^{*}$ & $67.2 \pm 10.0^{*}$ & $75.2 \pm 12.8^{*}$ & $8,977 \pm 2,164^{*}$ \\
\hline Completion of operation & $125.4 \pm 19.1^{*}$ & $68.8 \pm 12.6^{*}$ & $74.7 \pm 10.9^{*}$ & $9,360 \pm 1,977^{*}$ \\
\hline \multicolumn{5}{|c|}{ Hypertensive patients with preoperative oral nifedipine $(N=44)$} \\
\hline On arrival at the office & $182.1 \pm 13.8$ & $102.8 \pm 12.5$ & $87.2 \pm 17.1$ & $15,901 \pm 3,623$ \\
\hline Prior to sedation & $144.7 \pm 23.1^{*}$ & $77.3 \pm 15.9^{*}$ & $89.3 \pm 16.1$ & $12,986 \pm 3,437^{*}$ \\
\hline During operation & $119.8 \pm 16.5^{*}$ & $65.8 \pm 10.0^{*}$ & $78.0 \pm 12.3^{*}$ & $9,413 \pm 2,265^{*}$ \\
\hline Completion of operation & $130.1 \pm 18.8^{*}$ & $71.4 \pm 13.2^{*}$ & $78.4 \pm 13.6^{*}$ & $10,294 \pm 2,728^{*}$ \\
\hline
\end{tabular}


regular dental care, though a stress reduction protocol is necessary for stage I hypertension [2,7]. In accordance with the guidelines during oral surgery, the blood pressure of hypertensive patients should be maintained at a normal or prehypertension level. RPP is a reliable predictor of myocardial oxygen consumption [8], and RPP $>12,000 \mathrm{bpm} \times$ $\mathrm{mmHg}$ is associated with myocardial ischemia $[9,10]$. In this study, although blood pressure was managed by a physician, hypertensive patients showed SBP $>160 \mathrm{mmHg}$ when they visited the dental office for dental implant surgery, and $50 \%$ of hypertensive patients showed high RPP after 30 min of rest. In patients presenting with high blood pressure and high RPP, anxiety and fear must be reduced by conscious sedation and antihypertensives to prevent cardiovascular complications during dental implant surgery.

Increases in SBP due to psychological stress are proportional to age and baseline blood pressure [4]. Intravenous sedation stabilizes measurable changes in blood pressure and pulse rate due to fear and anxiety about dental treatment and has been used to manage patients with ischemic heart disease and hypertension [11]. In this study, the effect of intravenous sedation was as follows: SBP and RPP, compared with those prior to intravenous sedation, were decreased by $15 \%$ and $20 \%$ in patients with normal blood pressure, $15 \%$ and $25 \%$ in hypertensive patients without oral nifedipine, and $15 \%$ to $20 \%$ and $20 \%$ to $30 \%$ in hypertensive patients with administered nifedipine, respectively. That is, SBP and myocardial oxygen consumption of prehypertension and stage I hypertension can be reduced to the levels recommended for dental treatment before surgery by intravenous sedation.

For patients with stage 2 hypertension before operation, it is difficult to maintain the recommended blood pressure during surgery using only intravenous sedation, and it is necessary to decrease blood pressure by antihypertensive drugs. In this study, the blood pressure of patients with sustained hypertension was reduced to stage I hypertension about 30 min after administration of oral nifedipine. On the other hand, the decrease in RPP after oral nifedipine administration was not less than $12,000 \mathrm{bpm} \times \mathrm{mmHg}$, which could be due to the fact that an increase in pulse rate with nifedipine by reflex tachycardia. Thereafter, blood pressure and RPP during surgery under intravenous sedation has remained at levels similar to those of hypertensive patients with well-controlled blood pressure. Maximum effect (21.4\% decreases in SBP) appears in 30 to $60 \mathrm{~min}$ and lasts about $3 \mathrm{~h}$ on oral administration of nifedipine [12]. The half-lives of oral nifedipine, diltiazem and verapamil, and calcium antagonists are 0.2 to $1 \mathrm{~h}, 6$ to $8 \mathrm{~h}$, and 6 to $8 \mathrm{~h}$, respectively [13]. Since oral nifedipine has the properties of fast onset (30 to $45 \mathrm{~min}$ ) [14] and relatively short duration, it is suitable for outpatient dental implant surgery and is useful in perioperative management of patients with hypertension.
The overdose of vasoconstrictor that is added to the local anesthetic in order to prolong the anesthetic effect and hemostatic action may cause increased blood pressure and arrhythmias. Elevation of blood pressure in hypertensive patients is greater than that in normotensive patients during dental surgery [15]. Although there is an increase in blood pressure and tachycardia when using three cartridges of local anesthetic containing epinephrine $1: 10,000(5.4 \mathrm{ml})$, there are no adverse symptoms in patients with normal blood pressure [16]. Little recommended that the amount of local anesthetic solution administered should be less than two cartridges $(3.6 \mathrm{ml})$ for patients with hypertension [1]. Nakamura et al. reported that patients with essential hypertension who have been administered nifedipine can receive less than 3.6 cartridges of local anesthetic containing epinephrine 1:80,000 (6.4 ml) [17]. The administration of exogenous epinephrine with local anesthesia produces the highest plasma concentration in 3 to $6 \mathrm{~min}$ and lasts for $20 \mathrm{~min}$ [18]. It has been reported that the anesthetic rate of $2 \%$ lidocaine containing 1:10,000 epinephrine is $47 \%$ 45 min after administration and 27\% 60 min after administration [19]. During dental implant surgery which requires a relatively long duration and a wide field in patients with hypertension, administration of conduction anesthesia including inferior alveolar block and posterior superior alveolar nerve block is desirable. When the patient complains of pain, it is important to add local anesthesia while monitoring blood pressure to prevent increased blood pressure caused by pain.

Implant surgery is performed in patients with a wide age range, including elderly patients with hypertension. Dentists or oral surgeons often encounter hypertensive patients who are undiagnosed or noncompliant. Among Japanese over the age of $30,60 \%$ of men and $44.6 \%$ of women suffer from high blood pressure, and $33.8 \%$ of men and $25.6 \%$ of women with a history of hypertension have not been managed medically [20]. In this study, though 13 of the patients did not have a history of hypertension, they were diagnosed with essential hypertension by a physician because they had high blood pressure before surgery. Among patients with a history of high blood pressure, 31 patients (29\%) showed high blood pressure before surgery. Because there are many of dental patients with undiagnosed or noncompliant hypertension, blood pressure measurement before treatment, particularly invasive surgery, is indispensable.

For dental implant surgery in hypertensive patients who are not adequately controlled, the application of intravenous sedation and preoperative antihypertensive medication would be useful in order to prevent perioperative hypertension crisis including hypertension emergency with endorgan damage or hypertension urgency without end-organ damage. Since sublingual administration of immediate- 
release (IR) nifedipine may cause side effects such as significant decrease in blood pressure, reflex tachycardia, and acute myocardial infarction [21], the sublingual administration of IR nifedipine to hypertension crisis has not been approved by the Food and Drug Administration (1985) and Japanese Society of Hypertension Guidelines for the Management of Hypertension (2000). Since we could manage patients with high blood pressure without any cerebrovascular complications by oral administration of nifedipine under closely monitoring, it may be concluded that preoperative administration of oral nifedipine to patients with high blood pressure may be effective to prevent hypertensive crisis due to sudden rise in blood pressure during surgery. Further studies are necessary to evaluate the usefulness of captopril, clonidine, and labetalol, which have been reported as alternatives to nifedipine in emergency hypertension [22-24] in patients with high blood pressure.

\section{Conclusions}

In this study, we showed that the stable hemodynamic was obtained by performing intravenous sedation and oral administration of nifedipine for patients with hypertension. It is important not only to understand the systemic management of the patient but also to obtain stabled hemodynamic by performing intravenous sedation and oral administration of nifedipine for patients with hypertension in order to perform the implant surgery safely, and it could be possible to prevent the medical sequelae.

\begin{abstract}
Abbreviations
SBP: Systolic blood pressure; DBP: Diastolic blood pressure; PR: Pulse rate; RPP: Rate pressure product; ECG: Electrocardiogram; $\mathrm{SpO}_{2}$ : Percutaneous oxygen saturation; ANOVA: Analysis of variance; IR: Immediate-release.
\end{abstract}

\section{Competing interests}

Motoshi Kimura, Yoshihiro Takasugi, Shigeyoshi Hanano, Katsuyuki Terabe and Yuko Kimura declare that they have no competing interests.

\section{Authors' contributions}

YT and MK designed the study; MK, SH, and KT performed the surgeries; YT performed the intravenous sedation. YT, MK, and YK collected and analyzed the data; MK wrote the manuscript. YT revised the manuscript. All authors read and approved the final manuscript.

\section{Author details}

${ }^{1}$ Hanano Dental Clinic, 4-2-3 Yamanoue, Hirakata, Osaka 573-0047, Japan. ${ }^{2}$ Department of Anesthesiology, Kinki University Faculty of Medicine, 377-2 Ohno-Higashi, Osaka-Sayama, Osaka 589-8511, Japan. ${ }^{3}$ Terabe Dental Clinic, 4-249 Sakae-cho, Tsu, Mie 514-0004, Japan. ${ }^{4}$ First Department of Internal Medicine, Osaka Medical College, 2-7 Daigaku-machi, Takatsuki, Osaka 569-8686, Japan.

Received: 8 October 2014 Accepted: 14 January 2015

Published online: 18 March 2015

\section{References}

1. Little JW. The impact on dentistry of recent advances in the management of hypertension. Oral Surg Oral Med Oral Pathol Oral Radiol Endod. 2000:90:591-9.

2. Aubertin MA. The hypertensive patient in dental practice: updated recommendations for classification, prevention, monitoring, and dental management. Gen Dent. 2004;52:544-52.
3. Vaughan CJ, Delanty N. Hypertensive emergencies. Lancet. 2000;356:411-7.

4. Tsuchihashi T, Takata Y, Kurokawa H, Miura K, Maruoka Y, Kajiyama M, et al. Blood pressure response during dental surgery. Hypertens Res. 1996;19:189-94.

5. Ramsay MA, Savege TM, Simpson BR, Goodwin R. Controlled sedation with alphaxalone-alphadolone. Br Med J. 1974;2:656-9.

6. Chobanian AV, Bakris GL, Black HR, Cushman WC, Green LA, Izzo Jr JL, et al. The seventh report of the Joint National Committee on Prevention, Detection, Evaluation, and Treatment of High Blood Pressure: the JNC 7 report. JAMA. 2003;289:2560-72.

7. Holm SW, Cunningham Jr LL, Bensadoun E, Madsen MJ. Hypertension: classification, pathophysiology, and management during outpatient sedation and local anesthesia. J Oral Maxillofac Surg. 2006;64:111-21.

8. Gobel FL, Norstrom LA, Nelson RR, Jorgensen CR, Wang Y. The rate-pressure product as an index of myocardial oxygen consumption during exercise in patients with angina pectoris. Circulation. 1978;57:549-56.

9. White WB. Heart rate and the rate-pressure product as determinants of cardiovascular risk in patients with hypertension. Am J Hypertens. 1999;12:50S-5.

10. Urban MK, Gordon MA, Harris SN, O'Connor T, Barash PG. Intraoperative hemodynamic changes are not good indicators of myocardial ischemia. Anesth Analg. 1993;76:942-9.

11. Ichinohe T, Kaneko Y, Nakakuki T, Aida H, Abe H. Systemic management of dental patients with cardiovascular disease. Anesth Prog. 1989;36:219-21.

12. Kuwajima I, Ueda K, Kamata C, Matsushita S, Kuramoto K, Murakami M, et al. A study on the effects of nifedipine in hypertensive crises and severe hypertension. Jpn Heart J. 1978;19:455-67.

13. Elliott WJ, Ram CV. Calcium channel blockers. J Clin Hypertens (Greenwich). 2011;13:687-9.

14. Cohan JA, Checcio LM. Nifedipine in the management of hypertensive emergencies: report of two cases and review of the literature. Am J Emerg Med. 1985;3:524-30.

15. Abraham-Inpijn L, Borgmeijer-Hoelen A, Gortzak RAT. Changes in blood pressure, heart rate, and electrocardiogram during dental treatment with use of local anesthesia. J Am Dent Assoc. 1988;116:531-6.

16. Brand HS, Gortzak RA, Palmer-Bouva CC, Abraham RE, Abraham-Inpijn L. Cardiovascular and neuroendocrine responses during acute stress induced by different types of dental treatment. Int Dent J. 1995;45:45-8.

17. Nakamura $K$, Shionoya $Y$, Furuya $H$. The effect of a sublingually administered calcium antagonist, nifedipine, on the action of epinephrine in patients with essential hypertension. J J.jpn Dent Soc Anesthesiol. 1987;15:244-53 (In Japanese with English abstract).

18. Knoll-Köhler E, Knöller M, Brandt K, Becker J. Cardiohemodynamic and serum catecholamine response to surgical removal of impacted mandibular third molars under local anesthesia: a randomized double-blind parallel group and crossover study. J Oral Maxillofac Surg. 1991;49:957-62.

19. Katz S, Drum M, Reader A, Nusstein J, Beck M. A prospective, randomized, double-blind comparison of 2\% lidocaine with 1:100,000 epinephrine, 4\% prilocaine with 1:200,000 epinephrine, and 4\% prilocaine for maxillary infiltrations. Anesth Prog. 2010;57:45-51.

20. Ministry of Health, Labour and Welfare, Japan. National health and nutrition survey Japan, 2012. (in Japanese)

21. Grossman E, Messerli FH, Grodzicki T, Kowey P. Should a moratorium be placed on sublingual nifedipine capsules given for hypertensive emergencies and pseudoemergencies? JAMA. 1996;276:1328-31.

22. Thach AM, Schultz PJ. Nonemergent hypertension, new perspectives. Adv Updat Cardiovasc Emerg. 1995;13:1009-23.

23. Murphy C. Hypertensive emergencies. Adv Updat Cardiovasc Emerg. 1995;13:973-1007.

24. Hirschl M. Guidelines for the drug treatment of hypertensive crises. Drugs. 1995;50:991-1000. 